\title{
Antibiotic treatment impaired growth of tobacco in vitro shoots is associated with oxidative injury and reduced microbiome diversity
}

Inga Tamošiūnè

Lithuanian Research Centre for Agriculture and Forestry

Elena Andriūnaitè

Lithuanian Research Centre for Agriculture and Forestry

Jurgita Vinskienè

Lithuanian Research Centre for Agriculture and Forestry

Vidmantas Stanys

Lithuanian Research Centre for Agriculture and Forestry

Rytis Rugienius

Lithuanian Research Centre for Agriculture and Forestry

Danas Baniulis ( $\nabla$ danas.baniulis@lammc.lt)

Lithuanian Research Centre for Agriculture and Forestry

\section{Research Article}

Keywords: metagenomic analysis, Mycobacterium, Nicotiana tabacum, timentin

Posted Date: December 1st, 2021

DOI: https://doi.org/10.21203/rs.3.rs-1071558/v1

License: (9) (i) This work is licensed under a Creative Commons Attribution 4.0 International License.

Read Full License 


\section{Abstract}

Plant in vitro cultures initiated from surface-sterilized explants often harbor complex microbial community. Antibiotics are commonly used to decontaminate plant tissue culture or during genetic transformation, however, the effect of antibiotic treatment on the diversity of indigenous microbial population and consequences for performance of the tissue culture are not completely understood. Therefore, the aim of this study was to assess the effect of antibiotic treatment on the growth and stress level of tobacco (Nicotiana tabacum L.) in vitro shoots as well as the composition of plant-associated microbiome. The study revealed that shoot cultivation on medium supplemented with $250 \mathrm{mg} \mathrm{L}^{-1}$ timentin resulted in $29 \pm 4 \%$ reduced biomass accumulation and 1.2-1.6 fold higher level of oxidative stress injury compared to control. Moreover, the growth properties of shoots were only partially restored after transfer to medium without antibiotic. Shoot microbiome analysis using multi-variable region-based 16S rRNA sequencing revealed abundant microbial community in the control tobacco shoots, including bacteria from 59 families, however, it was largely dominated by Mycobacteriaceae. The antibiotic treatment resulted in a decline of species richness (the number of families was reduced 4.5-fold) and increased domination by the Mycobacteriaceae family. The results imply that the diversity of plantassociated microbiome might represent a significant factor contributing to the efficient propagation of in vitro tissue culture.

\section{Introduction}

Endophytes are a class of endosymbiotic microorganisms that inhabit the internal plant tissues ${ }^{1}$. Being protected inside explant or seed tissues, endophytic bacteria evade surface sterilization procedures used for cell culture initiation, and they are common in plant tissues grown in vitro ${ }^{2-4}$. Some bacterial endophytes maintain habitual concealed lifestyle or due to limited bacterial growth-supporting media conditions remain latent over extended periods of in vitro tissue cultivation. In fact, several studies showed a beneficial effect of endophytic bacteria on the growth of in vitro cultures of tomato ${ }^{5}$, grapevine ${ }^{6}$, sweet cherry ${ }^{7}$, apple ${ }^{8}$, purple coneflower ${ }^{9}$ or tobacco ${ }^{10}$. However, the formation of bacterial colonies on culture medium and bacterial overgrowth of plant tissues are rather common and manifest as infection by a variety of pathogenic species or the non-fastidious proliferation of commensal endophytic bacteria that can be triggered by the changes in environmental conditions or plant host physiology ${ }^{11-13}$.

To eliminate contamination of endophytic origin, pretreatment with high dose ${ }^{14}$ or medium supplementation with microbial growth-inhibiting ${ }^{15}$ antibiotics have been used and is often reported to improve the propagation or regenerative properties of the tissues $4,16-18$. Another common application of antibiotics for in vitro tissue culture is related to the use of Agrobacterium tumefaciens bacterium-to-plant DNA transfer machinery as an instrument in the plant genetic transformation ${ }^{19}$. Although alternative methods for the selection of genetically modified plants have been developed ${ }^{20}$, antibiotics remain indispensable for control of Agrobacterium growth upon plant tissue culture transformation. Left untreated, Agrobacterium, the causal agent of crown gall disease, shows excessive cell proliferation, that 
elicits a defense response in plant cells leading to a detrimental effect on plant tissue growth and efficiency of transformation ${ }^{21-23}$. Cephalosporin and penicillin-type antibiotics, such as cefotaxime, carbenicillin or timentin, active against gram-negative bacteria are commonly used ${ }^{24-26}$. In culture medium, carbenicillin and penicillins are broken down to physiologically active levels of the auxin-related compounds promoting organogenesis of explants ${ }^{27}$, however, at higher antibiotic concentration, proline accumulation, oxidative injury and reduced antioxidative activity have been reported indicating elevated stress level ${ }^{28}$. Previously, low cytotoxic effect of antibiotic timentin on in vitro plant tissues has been described 4,24 , and it is commonly used for transformation procedures in the range of concentrations from $150 \mathrm{mg} \mathrm{L}^{-124}$ to $500 \mathrm{mg} \mathrm{L}^{-129}$, sometimes in combination with lower concentrations of other antibiotics $^{30}$.

Cultivated tobacco (Nicotiana tabacum L.) has well established in vitro culture conditions ${ }^{31}$ and it has been commonly used as model species for genetic transformation studies as well as for practical application in molecular farming using in vitro techniques or contained greenhouse practice ${ }^{32-34}$. Recently, the composition of the endophytic microbiome of field-grown tobacco plants or seeds has been explored using cultivation and next-generation sequencing-based methods ${ }^{35,36}$ and several endophytic species of the Bacillus genus were isolated from tobacco leaves ${ }^{10,37}$. However, the microbial diversity of in vitro cultivated tobacco tissues had not been addressed so far.

Whereas antibiotics find application for explant and tissue culture decontamination or control of bacterial overgrowth during plant transformation, the effect of antibiotic treatment on the composition of endogenous microbial population in tissue culture and the long-term consequences on plant response to stress and growth parameters have not been completely understood. Considering the established role of endophytic bacteria, as modulator of plant growth and adaptation, it could be expected that the antibiotic-induced perturbation of the endophytic community might affect the efficiency of the in vitro culture propagation. Therefore, the aim of this study was to assess the effect of antibiotic timentin on growth, stress level of tobacco shoot in vitro culture and composition of plant-associated microbiome. Changes of shoot biomass accumulation and oxidative stress injury of cellular membranes during the antibiotic treatment and residual effect following the treatment were assessed. The composition of shoot bacterial population before and after the antibiotic treatment was investigated using a multi-variable $16 \mathrm{~S}$ rRNA region-based metagenomic analysis. To further refine taxonomic assignment of the dominant taxa that was revealed by the metagenomic analysis, isolate of Mycobacterium sp. was obtained using cultivation technique and its antibiotic resistance was tested.

\section{Results}

Effect of antibiotic on tobacco shoot growth and oxidative stress injury. Antibiotics could be cytotoxic to plant tissues at concentrations required for efficient control of the bacterium, usually in the range of 100 to $500 \mathrm{mg} \mathrm{I}^{-1} 38$. Although low cytotoxic effect of antibiotic timentin on in vitro plant tissues had been documented $4,24,29$ and it had been commonly used for plant cell culture at concentrations up to $500 \mathrm{mg}$ 
$\mathrm{L}^{-124,29}$, our experiments showed suppressed growth of tobacco in vitro shoots on medium supplemented with $250 \mathrm{mg} \mathrm{L}^{-1}$ timentin. After 3 weeks of cultivation, an average fresh weight (FW) of the control tobacco shoots (TC) was estimated at $164.7 \pm 3.3 \mathrm{mg}$, and FW of the shoots cultivated on medium containing timentin (TA) was reduced by $29 \pm 4 \%\left(p=1.5 \cdot 10^{-7}\right)$ compared to TC.

An analysis of the membrane lipid oxidative injury of the TC shoots revealed a significant variation of the MDA concentration during the three weeks of the propagation cycle (Fig. 1). The highest value was detected at day 4 after transfer to fresh medium and was estimated at $95.6 \pm 2.6 \mathrm{nmol} \cdot \mathrm{g}^{-1} \mathrm{FW}$. The increased MDA accumulation in the control shoots was likely a consequence of the combined effect of stress associated with tissue senescence and shoot injury during the transfer to the fresh medium which was followed by adaptation and active growth on the fresh medium resulting in $\sim 1.3$ fold $\left(p=4 \cdot 10^{-6}\right)$ decrease in MDA concentration at the end of the first week of cultivation (day 7), and subsequent gradual increase in MDA concentration during the course of culture senescence over the remaining two weeks of the propagation cycle. However, the TA shoots maintained on medium with antibiotic did not follow this cycle and showed 1.2-1.6 fold $(p<0,014)$ higher levels of MDA compared to the TC over the entire period of propagation cycle (Fig. 1) that could be a direct consequence of antibiotic-induced cytotoxic effect.

Interestingly, after transfer to the medium without antibiotic (PA), a consistent residual negative effect of antibiotic treatment on shoot growth vigor and stress level was observed for at least several passages used in the experiments. Cumulative data from several of these experiments revealed that growth vigor of the shoots was only partially restored and accumulation of biomass remained significantly lower $(9 \pm 3 \%$, $p=0.013$ ) compared to TC (Supporting Material Fig. S1). Similarly, the level of MDA accumulation was reduced as compared to TA and showed a significant decrease during the first week after transfer to fresh medium, nevertheless, it remained 1.1-1.3 $(p<0.033)$ fold higher compared to the control over the remaining two weeks of the propagation cycle.

Analysis of bacterial diversity in tobacco in vitro shoot samples. To investigate bacterial diversity in tobacco shoot culture and to assess the effect of antibiotic treatment on the bacterial diversity, DNA extracted from shoots of the TC and PA experimental groups was subjected to metagenomic analysis using the lon Torrent sequencing platform. Six different DNA extraction and enrichment methods were used for DNA preparation to assess capability to improve the efficiency of the bacterial 16S rRNA metagenomic analysis using as a template the DNA samples derived from the plastid and mitochondrial DNA rich plant material. The PCR amplicon libraries were generated using a multi-variable region approach comprising six regions of the 16S rRNA represented by a combination of five primer pairs (V2, V3, V4, V6-7 and V8) included in the 16S Metagenomic kit (Thermo-Fisher Scientific) (Supporting Material Fig. S2).

The overall number of high-quality mapped sequences with 224-225 bp read length was similar for the TC and PA libraries (1,027,576 and 1,175,938, respectively), but varied up to 3.5 -fold for different DNA preparation methods and primer pairs specific to distinct 16S rRNA gene regions (Supporting Material Table S1 and Fig. S3). The proportion of bacterial sequences varied from 10 to $29 \%$ for the different DNA 
extraction methods (Supporting Material Fig. S3A). Independent of the experimental group, consistently higher content of bacterial OTUs ( $>25 \%$ of all reads) was detected for samples prepared with DNA extraction methods described by Doyle ${ }^{39}$ and Ding et al. ${ }^{40}$. For the TC shoots, similar results were also obtained using DNA extraction method described by Li et al. ${ }^{41}$. The remaining two methods resulted in a consistently lower proportion of the bacterial OTUs. Bacterial DNA enrichment by selective organelle lysis using SDS and $\mathrm{NaCl}$ as previously described by Wang et al. ${ }^{42}$ for preparation of microbial metagenomic libraries of tropic tree Mallotus nudiflorus did not increase bacterial OTU content for the tobacco shoot samples as compared to other DNA extraction methods and the approach would require further optimization.

The proportion of sequences assigned to the bacterial and tobacco plastid or mitochondrial 16S rRNA also varied depending on PCR amplification region and/or specificity. Two primer pairs specific to the V4 and $\mathrm{V} 8$ regions generated the largest number of reads mapped as bacterial OTUs and the largest proportion of the bacterial OTUs as compared to the overall number of reads (Supporting Material Fig. S3B). Meanwhile, the overall amplification efficiency of the V6-7 and V2 primers was 2- to 4-fold lower, the resulting proportion of bacterial sequences was comparable to the $\mathrm{V} 8$. The $\mathrm{V} 3$ primers generated a large number of reads which was comparable to V8 but resulted in the lowest proportion of bacterial sequences. Previously, the V3 region had been shown to efficiently represent the diversity of microbial communities of the fecal or sewage samples ${ }^{43-45}$, however, our analysis using plant-derived samples showed and V3 primers included in the 16S Metagenomics Kit showed a strong predisposition toward plastid and/or mitochondrial sequences. It is notable that, for all primer sets, a consistently lower ratio of bacterial to plastid/mitochondrial OTUs was obtained for the PA as compared to the TC sample which could be a result of lower yields of bacterial DNA due to lower bacterial density in the antibiotic-treated shoot sample.

Principal coordinate analysis (PCoA) of the microbiome data sets using Bray-Curtis dissimilarity matrix represented variation mainly between the two tobacco shoot experimental groups on the first coordinate and variation resulting from the application of multi-variable region $16 \mathrm{~S}$ analysis were plotted mainly on the second coordinate (Fig. 2). Although larger variation was observed among the primer pairs for the PA sample, the overall pattern was very similar to that observed for the TC sample where V2 and V4 data sets represented the largest difference. The larger variation within the PA group was likely the result of a 5.6-fold lower number of OTUs mapped as compared to the TA sample (Supporting Material Table S1).

The region-specific primer capability to represent bacterial taxonomic diversity was assessed using rarefaction and alpha diversity analysis. A number of reads representing bacterial OTUs of the V3 data set were insufficient for the rarefaction analysis. For the remaining four regions the saturated curves indicated that the bacterial diversity was sufficiently represented and a several-fold difference in species richness between the two experimental groups, TC and TA, was revealed (Supporting Material Fig. S4). Alpha diversity analysis revealed a similar distribution of the Richness measure and the related Chao1 and Fisher's diversity indices (Supporting Material Table S2 and Fig. S5) among the data sets generated using distinct primer pairs. A significant difference was detected for the Evenness measure due to the 
high value estimated for the $\mathrm{V} 3$ region which appeared mainly related to the under-representation of the Mycobacteriaceae family in the V3 data set ( 0.2 and $40 \%$ of bacterial OTUs for the TC and PA, respectively) as compared to other primer pairs where the family was largely dominant $(79-90 \%$ and $94-$ $98 \%$, respectively) (Supporting Material Table S3; Fig. 4).

To evaluate 16S rRNA region-specific primer propensity for specific taxonomic groups, family-level data was used to avoid bias due to the limited accuracy of genus or species identification using short-read sequences which could lead to underestimation of the primer specificity. The association among the OTUs obtained using different primer pairs was mapped on the UpSet plot using cumulative data from both experimental groups (Fig. 3). Among the 59 family level OTUs, $22 \%$ were detected by all primer pairs and were represented by $96 \%$ or $70 \%$ of all bacterial reads when the dominant Mycobacteriaceae family was included or excluded from the analysis, respectively. No singleton families were detected using V2specific primers and 26 ( $42 \%$ of all families) singleton families were detected by the remaining four primer pairs, however, these included only $0.4 \%$ of the total number of reads mapped to bacterial OTUs. Chitinophagaceae and Sinobacteraceae (880 and 560 reads, respectively) were the most abundant singleton families detected using V4- and V8-specific primers, meanwhile, the remaining singletons included OTUs of relatively low abundance ( $<100$ reads). The results indicate that the multi-variable $16 \mathrm{~S}$ rRNA region-based metagenomic analysis approach is capable to provide higher taxonomic resolution of microbial diversity as compared to results obtained using individual regions, especially for the low abundance taxa.

Previously, a variation of informative power for the same 16S rRNA region-specific primers was demonstrated in the study with human intestinal or environmental microbiota samples ${ }^{45-47}$. In our study, the read abundance for the same OTU also varied considerably among the primer pairs (Fig. 4). For example, the most abundant Mycobacteriaceae family was represented by $37 \%, 18 \%$ and $39 \%$ of the total number of reads using V4, V6-7 and V8 primers, meanwhile, only $6 \%$ and $<1 \%$ of the total number of reads were assigned to the family for the $\mathrm{V} 2$ and $\mathrm{V} 3$ primer data sets, respectively. In another instance, the distribution of reads assigned to the Paenibacillaceae family among V3, V4 and V8 data sets was $15 \%, 38 \%$ and $44 \%$, respectively, meanwhile, V2 and V6-7 represented only $3 \%$ and $<1 \%$ of the total number of reads, respectively. Regardless of the apparent taxa-specific amplification efficiency among the primer pairs, the hierarchical cluster analysis of the data did not reveal consistent distribution of taxonomic groups among the primer data sets (Fig. 4), possibly due to variation of primer specificity at lower taxonomic level or the bias introduced by variation of the taxa abundance. Nevertheless, the results imply that cumulative data obtained from several primer pairs with different taxa-specific amplification efficiency has limited quantitative capacity to represent species abundance variation (species evenness) within a sample, however, this would not affect the quantitative estimate of inter-sample abundance variation for specific OTU.

Antibiotic effect on bacterial diversity in tobacco shoot culture. Although both, the TA and PA, samples had a similar overall number of reads and reads mapped to bacterial OTUs, the number of unique OTUs was reduced from 153 to 27 upon the antibiotic treatment (Supporting Material Table S1). The antibiotic 
effect resulted in $42 \%$ of total variability of the microbiome data between the TA and PA experimental groups as represented by the first coordinate of the PCoA (Fig. 3), and alpha diversity analysis showed a significant reduction in species richness and evenness upon the antibiotic treatment (Fig. 5).

Among the six phyla detected in the tobacco shoot microbiome, Actinobacteria was dominant in both experimental groups and was mainly represented by the order of Actinomycetales including 17 and 3 families for the TC and PA experimental groups, respectively (indicated in a blue color font in Fig. 4; Supporting Material Table S3). It is remarkable that Actinobacteria included the most prevalent family of Mycobacteriaceae, representing $81 \%$ and $98 \%$ of mapped bacterial reads for the TC and PA, respectively. Microbacteriaceae represent another antibiotic treatment enduring actinobacteria that became the second most abundant OTU with a relative abundance of $1.6 \%$ in the antibiotic-treated shoot sample, meanwhile, Propionibacteriaceae and Acidimicrobiaceae were detected only at the marginal level. For the latter OTU, sequence comparison showed $98 \%$ sequence similarity to Mycobacteriaceae which could also imply inaccuracy of the sequence assignment. A similar assumption could be drawn for the assignment of Thermolithobacteraceae family of the phylum Firmicutes. Meanwhile, Bacillaceae and Paenibacillaceae were the most abundant among the remaining four families of Firmicutes representing $3 \%$ of mapped bacterial reads in the control shoots. In addition, Staphylococcaceae and Streptococcaceae were detected at low abundance but by three primer pairs each. The abundance of all Firmicutes was largely reduced ( $<0.1 \%$ of mapped reads) upon the antibiotic treatment.

In the control shoots, $1.6 \%$ of mapped reads were assigned to 5 families of Bacteriodetes, mainly represented by Sphingobacteriaceae, Chitinophagaceae and Flavobacteriaceae, but all of them were undetectable in the antibiotic-treated sample. Among the Proteobacteria including $8 \%$ and $0.5 \%$ of mapped bacterial reads of the TC and PA, respectively, class Alpha-proteobacteria included 10 families of order Rhizobiales representing many well-known beneficial plant-associated bacteria ${ }^{48}$, and also Caulobacteraceae and Sphingomonadaceae families including common environmental bacteria ${ }^{49}$, 50 . In the antibiotic-treated shoots only Caulobacteraceae and Bradyrhizobiaceae were detected at 4- and 10fold reduced abundance level compared to control shoots, respectively. Interestingly, families Kopriimonadaceae, Rhodobacteraceae and Rhodospirillaceae including species of common environmental and aquatic bacteria were detected at low abundance only in the antibiotic-treated shoots.

The Desulfovibrionaceae family of class Deltaproteobacteria was detected at low but similar abundance in both, TC and PA, shoots. However, more proliferous Beta-proteobacteria and Gamma-proteobacteria (including 5 and 7 families, respectively) were also largely reduced upon the antibiotic treatment. Among the Beta-proteobacteria were notable Alcaligenaceae and Methylophilaceae which include nitrifying and methylotrophic environmental bacteria 51,52, Meanwhile families Enterobacteriaceae and Pseudomonadaceae of Gamma-proteobacteria represent numerous plant endophytes or pathogens ${ }^{53}$. In addition, two families of the PVC superphylum, Planctomycetes and Verrucomicrobia, which includes common soil and plant root-associated bacteria ${ }^{54}$ were both detected at low abundance in the control shoots. 
Isolation of Actinobacteria and antibiotic resistance assessment. To further characterize the actinobacteria dominant in the in vitro tobacco shoot culture, the extract of antibiotic-treated shoots was plated on the Loewenstein-Jensen growth medium. The isolate obtained from bright yellow colonies forming after 6-8 weeks of incubation showed 98\% identity to the M. cookii strain ATCC 49103 (GenBank accession NR_114661.1) ${ }^{55}$ based on the $1407 \mathrm{nt}$ fragment of 16S rRNA. The isolate also sustained similar growth properties on the Actinobacteria Isolation Agar which was used for later cultivation and antibiotic resistance test. The isolate showed resistance to timentin at the concentration used for the tobacco shoot treatment $\left(250 \mathrm{mg} \cdot \mathrm{L}^{-1}\right)$ and chloramphenicol at $30 \mathrm{mg} \mathrm{L}^{-1}$ (Supporting Material Fig. S6), however, the growth of the isolate was suppressed by rifampicin at $25 \mathrm{mg} \mathrm{L}^{-1}$ which could potentially be used for the mycobacterium elimination from the plant in vitro culture.

\section{Discussion}

An abundant microbial community of the control tobacco shoots was revealed by metagenomic analysis using a multi-variable 16S rRNA region approach and 153 OTUs representing 59 families of the bacteria domain were detected (Fig. 4). Bacterial endophytes are common in the in vitro cultures initiated from surface-sterilized plant dormant tissues, such as seeds or buds, which contain a complex community of endophytic bacteria. Previously, the study by Thomas et al. ${ }^{56}$ revealed a vast diversity of endophytic bacteria prevailing in grapevine field shoots (mainly Proteobacteria but also Actinobacteria, Firmicutes and several less abundant phyla) and their introduction to in vitro shoot culture was demonstrated. The occurrence of endophytic bacteria in seeds is also well documented ${ }^{57,58}$. Since the tobacco in vitro culture used in our study was initiated from surface-sterilized seeds, it is possible that numerous bacterial species survived the sterilization procedure in internal tissues or even on the surface of seeds. Endophytic species vertically transmitted through seed are closely associated with plant host and often play a significant role in the regulation of plant growth and development physiology. Previously, Enterobacteriaceae was detected as the predominant species of the endophyte community of tobacco seeds of the four distinct tobacco cultivars, and a genotype-specific signature was observed for Alphaproteobacteria ${ }^{36}$. In our study, among the bacteria identified in shoot microbiome, numerous species of Bacillales, Rhizobiales, Burkholderiales or Enterobacteriales could include plant endophytic bacteria adapted for vertical transmission and capable to inhabit seed tissues.

Alternatively, endospore or biofilm-forming bacteria are adapted to survive in extreme environments, many show resilience to sterilizing agents and would endure seed sterilization procedures. Such route could be adequate for soil bacteria such as Mycobacterium spp. which forms resilient endospores ${ }^{59}$, are capable to colonize plant tissues but have not been detected in seeds so far ${ }^{60,61}$. Similarly, species of Sphingomonadaceae are widely distributed in nature, having been isolated from many different land and water habitats, as well as from plant root systems and could form resilient biofilms which often are capable to survive sterilization conditions ${ }^{62}$. 
Despite the multi-variable region metagenomic analysis used in our study, it had limited capacity to reflect intra-sample abundance differences among the taxa. It appeared that the control tobacco shoot microbiome was largely dominated by Mycobacteriaceae, and likely one of its dominant species was shown to be closely related to Mycobacterium cookii. Mycobacteria are widely distributed in water and soil ${ }^{63}$, however, despite the fact that the genus Mycobacterium comprises nearly 200 species, so far the research has mainly focused on obligate pathogens and information about plant-associated mycobacteria is relatively scarce. Mycobacterium spp. have been isolated from the rhizosphere of tomato 64 and rice plants ${ }^{65}$. They have also been detected by sequencing-based analysis as endophytes of rice roots and stems ${ }^{61}$, wheat roots ${ }^{66}$, shoots of rock plant ${ }^{67}$ and buds of Scots pine ${ }^{68}$. Although previously, Mycobacterium spp. have not been described as tobacco endophyte, it might not represent an exception and might vary depending on cultivation conditions. In particular, endophytic or epiphytic colonization would be plausible for the plants cultivated on peat substrate considering that mycobacteria are abundant in peat as they are common inhabitants of sphagnum vegetation ${ }^{69}$, which was also one of the first described habitats for $M$. cookii ${ }^{70}$.

It is notable that previously Mycobacterium spp. have been detected as dominant species in sweet cherry in vitro shoots ${ }^{71}$ and tissue cultures of Scots pine ${ }^{72}$. Although in the latter case, some species of Mycobacterium have been reported to suppress undifferentiated Scots pine tissue growth and reduce the length of hypocotyls of seedlings, no negative effect has been observed for other species ${ }^{72}$. However, plant growth regulating properties have not been described for species related to M. cookii so far.

In our study, antibiotic timentin treatment had a detrimental effect on the endophytic community of tobacco in vitro shoots. The number of detected families was reduced $\sim 4.5$-fold and most of the remaining families had largely reduced counts. Interestingly, antibiotic treatment had little effect on the abundance of the dominant Mycobacteriaceae and Microbacteriaceae families (Supporting Material Table S3) as well as two Alpha-proteobacteria families, Caulobacteraceae and Bradyrhizobiaceae, that showed a modest reduction in relative abundance. Recently, the antibiotic effect on the diversity and structure of bacterial communities was described in the soil environment ${ }^{73}$. The study revealed that tetracycline addition could change the microbial community composition and relative abundance of bacteria due to a combination of growth suppressing or bactericidal effect of tetracycline and the induction of bacterial antibiotic resistance. In our study, the antibiotic resistance of the $M$. cookii related isolate supported the notion that increased domination of the Mycobacteriaceae family in the timentin treated shoots is a consequence of antibiotic resistance. Resistance to carboxypenicillins, such as ticarcillin, had been well documented for a variety of bacteria including actinobacterial taxa ${ }^{74}$. In addition, the resistance to clavulanic acid inhibitor would be required to ensure effective growth observed for the Mycobacterium isolate, which is relatively common among bacteria as well ${ }^{75}$. Resistance to antibiotics is also the most likely explanation for the unchanged or moderately decreased abundance of other bacterial taxa detected in the antibiotic-treated shoots, however, this would require further experimental confirmation. 
The antibiotic-induced perturbations in composition and/or interactions within the plant-associated microbial community of the tobacco shoots could be a significant factor contributing to the enduring negative effect on the growth and adaptive capacity of the plant tissue culture. Plant microbial community has a profound effect on the health of the host plant and reduced microbial diversity often leads to disease development ${ }^{76,77}$. It could be proposed that the antibiotic-induced perturbations in microbial community structure could eliminate pathogen suppressing antagonistic interactions and facilitate the spread of pathogenic microorganisms or disease susceptibility of the plants could be increased due to elevated stress level. However, the antibiotic-treated tobacco shoots did not show symptoms that could be attributed to microbial pathogenesis, such as distorted morphology or necrosis of tissues (Supporting information, Figure S1). Alternatively, a loss of mutualistic interaction with beneficial endophytic bacteria could contribute to reduced adaptation to in vitro conditions and inhibited shoot growth. Indeed, growth-promoting ${ }^{5,9}$ stress-reducing activity $6,8,10$ of bacteria on in vitro propagated plants have been reported previously. A large diversity of bacteria affected by the antibiotic treatment detected in our analysis would lead to an extensive list of potential candidates for the beneficial interaction, therefore, further studies, potentially including specific bacterial isolates or microbial consortia, would be required to refine the role of plant microbiome in modulation of in vitro culture growth and adaptation to the in vitro environment.

\section{Conclusion}

Our study revealed that the antibiotic timentin induced suppression of growth and elevated stress level of tobacco in vitro shoots is followed by enduring residual negative effect after shoot transfer to medium without antibiotic. The analysis of the control tobacco shoot microbiome detected an extensive bacterial community dominated by the Mycobacteriaceae family which potentially could originate from seeds used to initiate the in vitro culture and might include endophytic species closely associated with the plant host as well as contamination of resilient environmental bacteria. The antibiotic treatment-induced decline of species richness, increased domination of Mycobacteriaceae and several other families were likely associated with antibiotic resistance traits of the bacteria. It was proposed that antibiotic-induced perturbation of shoot microbiome composition and/or interactions might contribute to the reduced adaptive capacity and impede the growth of the tobacco shoots leading to the reduced efficiency of the in vitro culture propagation. Therefore, further insights into the specific role of the members and interactions of the tobacco shoot microbiome would ensure a better understanding of the antibiotic effect on the in vitro tissue as holobiont and would potentially provide solutions required to improve the efficacy of the in vitro tissue culture.

\section{Methods}

Tobacco shoot culture in vitro and antibiotic treatment. Cultivated tobacco (Nicotiana tabacum cv. Samsun-NN) in vitro shoot culture was derived from seeds kindly provided by Prof. J.P.T. Valkonen (University of Helsinki). Plants were cultivated and all experiments were carried out in accordance with 
relevant guidelines. Tobacco shoots cultivated in vitro for over 5 years were used as a control (TC) and were maintained on solid in Murashige-Skoog (MS) medium ${ }^{78}$, supplemented with $0.75 \mathrm{mg} \cdot \mathrm{L}^{-1} 6$ benzylaminopurine, $30 \mathrm{~g} \cdot \mathrm{L}^{-1}$ sucrose, $0.8 \%$ agar at $25 \pm 1{ }^{\circ} \mathrm{C}$, under $150 \mu \mathrm{mol} \cdot \mathrm{m}^{-2} \cdot \mathrm{s}^{-1}$ intensity illumination of $16 \mathrm{~h}$ photoperiod. Antibiotic-treated tobacco shoots (TA) were maintained as described for the TC, except the medium was supplemented with timentin at $250 \mathrm{mg} \cdot \mathrm{L}^{-1}$. To ascertain stable and homogeneous physiological response in the shoot culture, the duration of the treatment was 6 months. Prolonged cultivation was also used to clear residual DNA from antibiotic-inactivated bacteria to avoid false-positive results during the shoot microbiome analysis based on metagenomic DNA sequencing. After transfer to medium without antibiotic (PA), the shoots were maintained under the same conditions as TC and at least one culture passage (one-month duration) was used before collecting samples for the analysis.

Samples for shoot fresh weight (FW) and microbial composition analysis were collected three weeks after transfer to fresh medium, and oxidative stress injury was assessed at four distinct time points during the propagation cycle.

Assessment of oxidative stress injury. Oxidative injury of tobacco shoot cellular membranes was estimated based on quantitative analysis of the lipid peroxidation product malondialdehyde (MDA) using previously described method ${ }^{79}, 80$. Homogenized frozen tobacco shoot powder was extracted with $50 \mathrm{mM}$ Tris- $\mathrm{HCl} \mathrm{pH} \mathrm{7.4,} \mathrm{containing} 1.5 \%$ of polyvinylpolypyrrolidone for $30 \mathrm{~min}$ at $4^{\circ} \mathrm{C}$ and centrifuged at 10,000 -g for 15 min at $4^{\circ} \mathrm{C}$. Equal amounts of tissue extract and $0.5 \%$ thiobarbituric acid in $20 \%$ trichloroacetic acid were mixed, heated at $95^{\circ} \mathrm{C}$ for $30 \mathrm{~min}$, cooled on ice and centrifuged at $10,000 \cdot \mathrm{g}$ for $5 \mathrm{~min}$. The absorbance measured at $532 \mathrm{~nm}$ was corrected by subtracting the absorbance value at $600 \mathrm{~nm}$ and MDA concentration was estimated using $\varepsilon=155 \mathrm{mM}^{-1} \mathrm{~cm}^{-1}$. The absence of interference from the absorbance of anthocyanins at $532 \mathrm{~nm}$ was verified using control samples without thiobarbituric acid.

Bacterial 16S RNA metagenomic analysis. The metagenomic analysis samples from the TC and PA experimental groups were fresh frozen in liquid $\mathrm{N}_{2}$ and stored at $-70^{\circ} \mathrm{C}$. To assess methods most efficient for bacterial DNA extraction from plant material, six different DNA extraction methods described by 39,40 , 81, 82, PureLink Microbiome DNA Purification kit (Thermo Fisher Scientific, USA) and a method for bacterial DNA enrichment using SDS detergent extraction ${ }^{42}$ were used. DNA libraries were prepared using five primer pairs from the lon 16S Metagenomics kit (Thermo Fisher Scientific, USA) (Supporting Material Fig. S2) as described previously ${ }^{83}$. Equal volumes of all DNA library samples (adjusted to $10 \mathrm{pM}$ ) were combined and emulsion PCR was carried out using lon OneTouch 2 System and lon PGM Hi-Q View OT2 Kit (Thermo Fisher Scientific, USA). The clonal libraries were enriched using lon OneTouch ES (Thermo Fisher Scientific, USA) and sequencing was performed using lon 316 v. 2 chip on the lon Personal Genome Machine system using lon PGM Hi-Q Sequencing kit (Thermo Fisher Scientific, USA). Base-calling and run demultiplexing were performed by Torrent Suite v.5.0.5 (Thermo Fisher Scientific, USA) with default parameters. Sequencing data were processed using $16 \mathrm{~S}$ Metagenomic workflow of the lon Reporter Software v.5.10.5.0 (Thermo Fisher Scientific, USA). Reads were trimmed by primers at both ends. The 
threshold for unique reads was set to 10. Taxonomic identification was performed using MicroSEQ 16S Reference Library v.2013.1 and Greengenes v.13.5 databases and N. tabacum plastid 16S rRNA (NC_001879.2:102762-104252) and mitochondrion 18S rRNA (NC_006581.1:108334-110235) sequences were included to detect sequences resulting from contamination of the plant mitochondrial and plastid host DNA. The threshold value for percentage identity for genus and species ID was $97 \%$ and $99 \%$, respectively.

Mycobacterium isolation, identification and antibiotic resistance test. Tobacco shoots were homogenized with a razor blade in MS medium, the homogenate was applied to Loewenstein-Jensen medium prepared from TB-Medium base (Merck, Germany) and incubated at room temperature for 6-8 weeks. The bacteria isolate was maintained on the Actinobacteria Isolation Agar (AIA). Bacterial DNA was isolated using the GeneJET Genomic DNA Purification kit (Thermo Fisher Scientific, USA). The 16S rRNA gene fragment was amplified using the universal primers as described previously ${ }^{10}$ and the sequence was queried at the NCBI BLAST server ${ }^{84}$. Isolate resistance to antibiotics was tested by cultivation on AIA medium supplemented with timentin $\left(250 \mathrm{mg} \cdot \mathrm{L}^{-1}\right)$, chloramphenicol $\left(30 \mathrm{mg} \cdot \mathrm{L}^{-1}\right)$ or rifampicin $\left(25 \mathrm{mg} \cdot \mathrm{L}^{-1}\right)$.

Statistical data analysis. Statistically significant differences of shoot FW and MDA accumulation were assessed by ANOVA analysis and Tukey post hoc test using IBM SPSS Statistics v.21 (IBM Inc.). Data are presented as the mean of at least 3 independent experiments and standard error of the mean.

Statistical analysis of the metagenomic data and visualization was performed using PCoA, Rarefaction, Alpha diversity and HeatMap functions of the Calypso web-based platform ${ }^{85}$. Total sum normalization was applied to taxonomic counts data by dividing feature read counts by the total number of reads in each sample. To account for the non-normal distribution of the data, cumulative-sum scaling and log2 transformation were used ${ }^{86}$. The representation of the microbiome by the primer pairs representing distinct rRNA regions was visualized using UpSet graph ${ }^{87}$.

\section{Declarations}

\section{Acknowledgements}

This project has received funding from European Regional Development Fund (project No. 01.2.2-LMT-K718-01-0037) under grant agreement with the Research Council of Lithuania (LMTLT). The authors would like to thank Dr. D. Gelvonauskienè, Dr. P. Haimi for assistance and constructive discussions and Ms. Z. Kajatienè for valuable technical support.

\section{Author Contributions}

I.T., E.A., R.R., V.S., D.B. conceptualized and designed the experiments; I.T., E.A., J.V., R.R. acquired, analyzed and interpreted the data; I.T., E.A., D.B. drafted the manuscript; R.R., V.S. contributed to critical revision of the manuscript. All authors reviewed the manuscript. 
Competing Interests: The authors declare no competing interests

\section{References}

1. Brader, G. et al. Ecology and genomic insights into plant-pathogenic and plant-nonpathogenic endophytes. Annu. Rev. Phytopathol, 55, 61-83 (2017).

2. Kukkurainen, S. et al. Occurrence and location of endophytic bacteria in garden and wild strawberry. HortScience, 40, 348-352 (2005).

3. Abreu-Tarazi, M. F. et al. Endophytic bacteria in long-term in vitro cultivated "axenic" pineapple microplants revealed by PCR-DGGE. World J. Microbiol. Biotechnol, 26, 555-560 (2010).

4. Leone, G. F. et al. Use of antibiotics to control endophytic bacterial growth migration onto culture medium in Eucalyptus cloeziana F. Muell.: a micropropagation approach. In Vitro Cell. Dev. Biol. Plant, 55, 421-432 (2019).

5. Botta, A. L., Santacecilia, A., Ercole, C., Cacchio, P. \& Del Gallo, M. vitro and in vivo inoculation of four endophytic bacteria on Lycopersicon esculentum. N. Biotechnology, 30, 666-674 (2013).

6. Salomon, M. V. et al. Bacteria isolated from roots and rhizosphere of Vitis vinifera retard water losses, induce abscisic acid accumulation and synthesis of defense-related terpenes in in vitro cultured grapevine. Physiol. Plant, 151, 359-374 (2014).

7. Quambusch, M., Brümmer, J., Haller, K., Winkelmann, T. \& Bartsch, M. Dynamics of endophytic bacteria in plant in vitro culture: quantification of three bacterial strains in Prunus avium in different plant organs and in vitro culture phases. Plant Cell Tiss. Org. Cult, 126, 305-317 (2016).

8. Tamosiune, I. et al. Endophytic Bacillus and Pseudomonas spp. modulate apple shoot growth, cellular redox balance, and protein expression under in vitro conditions. Front. Plant Sci, 9, 889 (2018).

9. Maggini, V. et al. Tissue specificity and differential effects on in vitro plant growth of single bacterial endophytes isolated from the roots, leaves and rhizospheric soil of Echinacea purpurea. BMC Plant Biol, 19, 284-284 (2019).

10. Andriūnaitè, E. et al. Stimulation of Nicotiana tabacum L. in vitro shoot growth by endophytic Bacillus cereus group bacteria. Microorganisms, 9, 1893 (2021).

11. Cassells, A. C.. Problems in tissue culture: culture contamination, in Micropropagation: Technology and Application. (eds. P.C. Debergh \& R.H. Zimmerman)31-44(Springer Netherlands, Dordrecht; 1991)

12. Reed, B. M., Buckley, P. M. \& DeWilde, T. N. Detection and eradication of endophytic bacteria from micropropagated mint plants. In Vitro Cell. Dev. Biol. Plant, 31, 53-57 (1995).

13. Cassells, A. C. \& Tahmatsidou, V. The influence of local plant growth conditions on non-fastidious bacterial contamination of meristem-tips of Hydrangea cultured in vitro. Plant Cell Tiss. Org. Cult, 47, 15-26 (1996). 
14. Kulkarni, A. A., Kelkar, S., Watve, M. \& Krishnamurthy, K. Characterization and control of endophytic bacterial contaminants in in vitro cultures of Piperspp., Taxus baccata subsp. wallichiana, and Withania somnifera. Can. J. Microbiol. 53, 63-74 (2007)

15. Thomas, P. vitro decline in plant cultures: detection of a legion of covert bacteria as the cause for degeneration of long-term micropropagated triploid watermelon cultures. Plant Cell Tiss. Org. Cult, 77, 173-179 (2004).

16. Pirttilä, A. M., Podolich, O., Koskimäki, J. J., Hohtola, E. \& Hohtola, A. Role of origin and endophyte infection in browning of bud-derived tissue cultures of Scots pine (Pinus sylvestris L.). Plant Cell Tiss. Org. Cult, 95, 47-55 (2008).

17. Thomas, P., Swarna, G. K., Patil, P. \& Rawal, R. D. Ubiquitous presence of normally non-culturable endophytic bacteria in field shoot-tips of banana and their gradual activation to quiescent cultivable form in tissue cultures. Plant Cell Tiss. Org. Cult, 93, 39-54 (2008).

18. Ray, S. S., Ali, M. N., Mukherjee, S., Chatterjee, G. \& Banerjee, M. Elimination and molecular identification of endophytic bacterial contaminants during in vitro propagation of Bambusa balcooa. World J. Microbiol. Biotechnol, 33, 1-9 (2017).

19. Anami, S., Njuguna, E., Coussens, G., Aesaert, S. \& Van Lijsebettens, M. Higher plant transformation: principles and molecular tools. Int. J. Dev. Biol, 57, 483-494 (2013).

20. Breyer, D., Kopertekh, L. \& Reheul, D. Alternatives to antibiotic resistance marker genes for in vitro selection of genetically modified plants - scientific developments, current use, operational access and biosafety considerations. Crit. Rev. Plant Sci, 33, 286-330 (2014).

21. Pu, X. \& Goodman, R. N. Induction of necrogenesis by Agrobacterium tumefaciens on grape explants. Physiol. Mol. Plant Pathol, 41, 241-254 (1992).

22. Deng, W., Pu, X. A., Goodman, R. N., Gordon, M. P. \& Nester, E. W. T-DNA genes responsible for inducing a necrotic response on grape vines. Mol. Plant-Microbe Interact, 8, 538-548 (1995).

23. Ozawa, K. Establishment of a high efficiency Agrobacterium-mediated transformation system of rice (Oryza sativa L.). Plant Sci, 176, 522-527 (2009).

24. Nauerby, B., Billing, K. \& Wyndaele, R. Influence of the antibiotic timentin on plant regeneration compared to carbenicillin and cefotaxime in concentrations suitable for elimination of Agrobacterium tumefaciens. Plant Sci, 123, 169-177 (1997).

25. Ogawa, Y. \& Mii, M. Screening for highly active $\beta$-lactam antibiotics against Agrobacterium tumefaciens. Arch. Microbiol, 181, 331-336 (2004).

26. Priya, A. M., Pandian, S. K. \& Manikandan, R. The effect of different antibiotics on the elimination of Agrobacterium and high frequency Agrobacterium-mediated transformation of indica rice (Oryza sativa L.). Czech J. Genet. Plant Breed, 48, 120-130 (2012).

27. Grzebelus, E. \& Skop, L. Effect of $\beta$-lactam antibiotics on plant regeneration in carrot protoplast cultures. In Vitro Cell. Dev. Biol. Plant, 50, 568-575 (2014).

28. Qin, Y. H., Teixeira da Silva, J. A., Bi, J. H., Zhang, S. L. \& Hu, G. B. Response of in vitro strawberry to antibiotics. Plant Growth Regul, 65, 183-193 (2011). 
29. Cheng, Z. M., Schnurr, J. A. \& Kapaun, J. A. Timentin as an alternative antibiotic for suppression of Agrobacterium tumefaciens in genetic transformation. Plant Cell Rep, 17, 646-649 (1998).

30. Kumar, R., Mamrutha, H. M., Kaur, A. \& Grewal, A. Synergistic effect of cefotaxime and timentin to suppress the Agrobacterium overgrowth in wheat (Triticum aestivum L.) transformation. Asian J. Microbiol., Biotechnol. Environ. Sci, 19, 961-967 (2017).

31. Topping, J. F. \& Lindsey, K. Shoot cultures and root cultures of tobacco, in Plant Tissue Culture Manual. (ed. K. Lindsey)67-79(Springer Netherlands, Dordrecht; 1991)

32. Colgan, R. et al. Optimisation of contained Nicotiana tabacum cultivation for the production of recombinant protein pharmaceuticals. Transgenic Res, 19, 241-256 (2010).

33. Tremblay, R., Wang, D., Jevnikar, A. M. \& Ma, S. Tobacco, a highly efficient green bioreactor for production of therapeutic proteins. Biotechnol. Adv, 28, 214-221 (2010).

34. Moustafa, K., Makhzoum, A. \& Tremouillaux-Guiller, J. Molecular farming on rescue of pharma industry for next generations. Crit. Rev. Biotechnol, 36, 840-850 (2016).

35. Zhou, J. et al. Characterization of the core microbiome in tobacco leaves during aging. Microbiologyopen, 9, e984-e984 (2020).

36. Chen, X. et al. Nicotiana tabacum seed endophytic communities share a common core structure and genotype-specific signatures in diverging cultivars. Comput. Struct. Biotechnol. J, 18, 287-295 (2020).

37. Chen, Z. et al. Species diversity characteristics of endophytic bacteria in tobacco at different regions of Yunnan Province. SW. China J. Agric. Sci, 28, 857-861 (2015).

38. Wojtania, A., PvBawska, J. \& Gabryszewska, E. Identification and elimination of bacterial contaminants from Pelargonium tissue cultures. J. Fruit Ornam. Plant Res, 13, 101-108 (2005).

39. Doyle, J. DNA Protocols for Plants, in Molecular Techniques in Taxonomy. (eds. G.M. Hewitt, A.W.B. Johnston \& J.P.W. Young)283-293(Springer Berlin Heidelberg, Berlin, Heidelberg; 1991)

40. Ding, T., Palmer, M. W. \& Melcher, U. Community terminal restriction fragment length polymorphisms reveal insights into the diversity and dynamics of leaf endophytic bacteria. BMC Microbiol, 13, 1 (2013).

41. Li, H., Luo, J., Hemphill, J. K., Wang, J. T. \& Gould, J. H. A rapid and high yielding DNA miniprep for cotton (Gossypium spp.). Plant Mol. Biol. Rep, 19, 183-183 (2001).

42. Wang, H. X., Geng, Z. L., Zeng, Y. \& Shen, Y. M. Enriching plant microbiota for a metagenomic library construction. Environ. Microbiol, 10, 2684-2691 (2008).

43. Milani, C. et al. Assessing the fecal microbiota: an optimized ion torrent $16 \mathrm{~S}$ rRNA gene-based analysis protocol. PLoS One, 8, e68739 (2013).

44. Cai, L., Ye, L., Tong, A. H. Y., Lok, S. \& Zhang, T. Biased diversity metrics revealed by bacterial 16S pyrotags derived from different primer sets. PLoS One, 8, e53649 (2013).

45. Aloisio, I. et al. Evaluation of the effects of intrapartum antibiotic prophylaxis on newborn intestinal microbiota using a sequencing approach targeted to multi hypervariable 16S rDNA regions. Appl. 
Microbiol. Biotechnol, 100, 5537-5546 (2016).

46. Winand, R. et al. Targeting the $16 \mathrm{~S}$ rRNA gene for bacterial identification in complex mixed samples: comparative evaluation of second (illumina) and third (oxford nanopore technologies) generation sequencing technologies. Int. J. Mol. Sci, 21, 298 (2020).

47. Torrell, H. et al. Multiomic approach to analyze infant gut microbiota: experimental and analytical method optimization. Biomolecules, 11, 999 (2021).

48. Erlacher, A. et al. Rhizobiales as functional and endosymbiontic members in the lichen symbiosis of Lobaria pulmonaria L. Front. Microbiol, 6, 53 (2015).

49. Abraham, W. R., Rohde, M. \& Bennasar, A. The family Caulobacteraceae, in The Prokaryotes: Alphaproteobacteria and Betaproteobacteria. (eds. E. Rosenberg, E.F. DeLong, S. Lory, E. Stackebrandt \& F. Thompson)179-205(Springer Berlin Heidelberg, Berlin, Heidelberg; 2014)

50. Glaeser, S. P. \& Kämpfer, P. The family Sphingomonadaceae, in The Prokaryotes: Alphaproteobacteria and Betaproteobacteria. (eds. E. Rosenberg, E.F. DeLong, S. Lory, E. Stackebrandt \& F. Thompson)641-707(Springer Berlin Heidelberg, Berlin, Heidelberg; 2014)

51. Velusamy, K. \& Krishnani, K. K. Heterotrophic nitrifying and oxygen tolerant denitrifying bacteria from greenwater system of coastal aquaculture. Appl. Biochem. Biotechnol, 169, 1978-1992 (2013).

52. Doronina, N., Kaparullina, E. \& Trotsenko, Y. The family Methylophilaceae, in The Prokaryotes: Alphaproteobacteria and Betaproteobacteria. (eds. E. Rosenberg, E.F. DeLong, S. Lory, E. Stackebrandt \& F. Thompson)869-880(Springer Berlin Heidelberg, Berlin, Heidelberg; 2014)

53. Torres, A. R. et al. Diversity of endophytic enterobacteria associated with different host plants. J. Microbiol, 46, 373-379 (2008).

54. Bünger, W., Jiang, X., Müller, J., Hurek, T. \& Reinhold-Hurek, B. Novel cultivated endophytic Verrucomicrobia reveal deep-rooting traits of bacteria to associate with plants. Sci. Rep, 10, 8692 (2020).

55. Turenne, C. Y., Tschetter, L., Wolfe, J. \& Kabani, A. Necessity of quality-controlled 16S rRNA gene sequence databases: Identifying nontuberculous Mycobacterium species. J. Clin. Microbiol, 39, 3637-3648 (2001).

56. Thomas, P., Sekhar, A. C. \& Shaik, S. P. High taxonomic diversity of cultivation-recalcitrant endophytic bacteria in grapevine field shoots, their in vitro introduction, and unsuspected persistence., 246, 879898 (2017).

57. Johnston-Monje, D. \& Raizada, M. N. Conservation and diversity of seed associated endophytes in Zea across boundaries of evolution, ethnography and ecology. PLoS One, 6, e20396 (2011).

58. Shahzad, R., Khan, A. L., Bilal, S., Asaf, S. \& Lee, I. J. What is there in seeds? Vertically transmitted endophytic resources for sustainable improvement in plant growth. Front. Plant Sci, 9, 24 (2018).

59. Fedrizzi, T. et al. Genomic characterization of Nontuberculous Mycobacteria. Sci. Rep, 7, 45258 (2017). 
60. Leff, J. W., Lynch, R. C., Kane, N. C. \& Fierer, N. Plant domestication and the assembly of bacterial and fungal communities associated with strains of the common sunflower, Helianthus annuus. New Phytol, 214, 412-423 (2017).

61. Wang, W. F., Zhai, Y. Y., Cao, L. X., Tan, H. M. \& Zhang, R. D. Illumina-based analysis of core actinobacteriome in roots, stems, and grains of rice. Microbiol. Res, 190, 12-18 (2016).

62. de Vries, H. J. et al. Isolation and characterization of Sphingomonadaceae from fouled membranes. npj Biofilms and Microbiomes, 5, 6 (2019).

63. Walsh, C. M., Gebert, M. J., Delgado-Baquerizo, M. \& Maestre, F. T. Fierer, N. A global survey of Mycobacterial diversity in soil. Appl. Environ. Microbiol, 85, e01180-01119 (2019).

64. Bouam, A., Armstrong, N., Levasseur, A. \& Drancourt, M. Mycobacterium terramassiliense, Mycobacterium rhizamassiliense and Mycobacterium numidiamassiliense sp. nov., three new Mycobacterium simiae complex species cultured from plant roots. Sci. Rep, 8, 9309 (2018).

65. Mano, H., Tanaka, F., Nakamura, C., Kaga, H. \& Morisaki, H. Culturable endophytic bacterial flora of the maturing leaves and roots of rice plants (Oryza sativa) cultivated in a paddy field. Microbes Environ, 22, 175-185 (2007).

66. Conn, V. M. \& Franco, C. M. M. Analysis of the endophytic actinobacterial population in the roots of wheat (Triticum aestivum L.) by terminal restriction fragment length polymorphism and sequencing of 16S rRNA clones. Appl. Environ. Microbiol, 70, 1787-1794 (2004).

67. Koskimäki, J. J., Hankala, E., Suorsa, M., Nylund, S. \& Pirttilä, A. M. Mycobacteria are hidden endophytes in the shoots of rock plant [Pogonatherum paniceum (Lam.) Hack.] (Poaceae). Environ. Microbiol. Rep, 2, 619-624 (2010).

68. Pirttilä, A. M., Pospiech, H., Laukkanen, H., Myllylä, R. \& Hohtola, A. Seasonal variations in location and population structure of endophytes in buds of Scots pine. Tree Physiol, 25, 289-297 (2005).

69. Kazda, J.. Mycobacteria as habitat microorganisms of sphagnum bog vegetation, in The Ecology of Mycobacteria. (ed. J. Kazda)32-36(Springer Netherlands, Dordrecht; 2000)

70. Kazda, J. et al. Mycobacterium cookii sp. nov. Int. J. Syst. Bacteriol, 40, 217-223 (1990).

71. Quambusch, M., Pirttilä, A. M., Tejesvi, M. V., Winkelmann, T. \& Bartsch, M. Endophytic bacteria in plant tissue culture: differences between easy- and difficult-to-propagate Prunus avium genotypes. Tree Physiol, 34, 524-533 (2014).

72. Laukkanen, H., Soini, H., Kontunen-Soppela, S., Hohtola, A. \& Viljanen, M. A mycobacterium isolated from tissue cultures of mature Pinus sylvestris interferes with growth of Scots pine seedlings. Tree Physiol, 20, 915-920 (2000).

73. Zheng, J. Y. et al. The effects of tetracycline residues on the microbial community structure of tobacco soil in pot experiment. Sci. Rep, 10, 8804 (2020).

74. Fatahi-Bafghi, M. Antibiotic resistance genes in the Actinobacteria phylum. Eur. J. Clin. Microbiol. Infect. Dis, 38, 1599-1624 (2019). 
75. Chaïbi, E. B., Sirot, D., Paul, G. \& Labia, R. Inhibitor-resistant TEM ß-lactamases: phenotypic, genetic and biochemical characteristics. J. Antimicrob. Chemother, 43, 447-458 (1999).

76. Berg, G. et al. Plant microbial diversity is suggested as the key to future biocontrol and health trends. FEMS Microbiol. Ecol, 93, fix050 (2017).

77. Sessitsch, A. \& Mitter, B. 21st century agriculture: integration of plant microbiomes for improved crop production and food security. Microb Biotechnol, 8, 32-33 (2015).

78. Murashige, T. \& Skoog, F. A revised medium for rapid growth and bio assays with tobacco tissue cultures. Physiol. Plant, 15, 473-497 (1962).

79. Hodges, D. M., DeLong, J. M., Forney, C. F. \& Prange, R. K. Improving the thiobarbituric acid-reactivesubstances assay for estimating lipid peroxidation in plant tissues containing anthocyanin and other interfering compounds., 207, 604-611 (1999).

80. Jagendorf, A. T. \& Takabe, T. Inducers of glycinebetaine synthesis in barley. Plant Physiol, 127, 1827-1835 (2001).

81. Li, J. H., Wang, E. T., Chen, W. F. \& Chen, W. X. Genetic diversity and potential for promotion of plant growth detected in nodule endophytic bacteria of soybean grown in Heilongjiang province of China. Soil Biol. Biochem, 40, 238-246 (2008).

82. Akinsanya, M. A., Goh, J. K., Lim, S. P. \& Ting, A. S. Y. Metagenomics study of endophytic bacteria in Aloe vera using next-generation technology. Genom. Data, 6, 159-163 (2015).

83. Tamosiune, I. et al. Cold plasma treatment of sunflower seeds modulates plant-associated microbiome and stimulates root and lateral organ growth. Front. Plant Sci, 11, 568924 (2020).

84. Madden, T. L., Tatusov, R. L. \& Zhang, J. Applications of network BLAST server. Methods Enzymol, 266, 131-141 (1996).

85. Zakrzewski, M. et al. Calypso: a user-friendly web-server for mining and visualizing microbiomeenvironment interactions., 33, 782-783 (2017).

86. Paulson, J. N., Stine, O. C., Bravo, H. C. \& Pop, M. Differential abundance analysis for microbial marker-gene surveys. Nat. Methods, 10, 1200-1202 (2013).

87. Lex, A., Gehlenborg, N., Strobelt, H., Vuillemot, R. \& Pfister, H. UpSet: visualization of intersecting sets. IEEE Trans. Vis. Comput. Graphics, 20, 1983-1992 (2014).

\section{Figures}




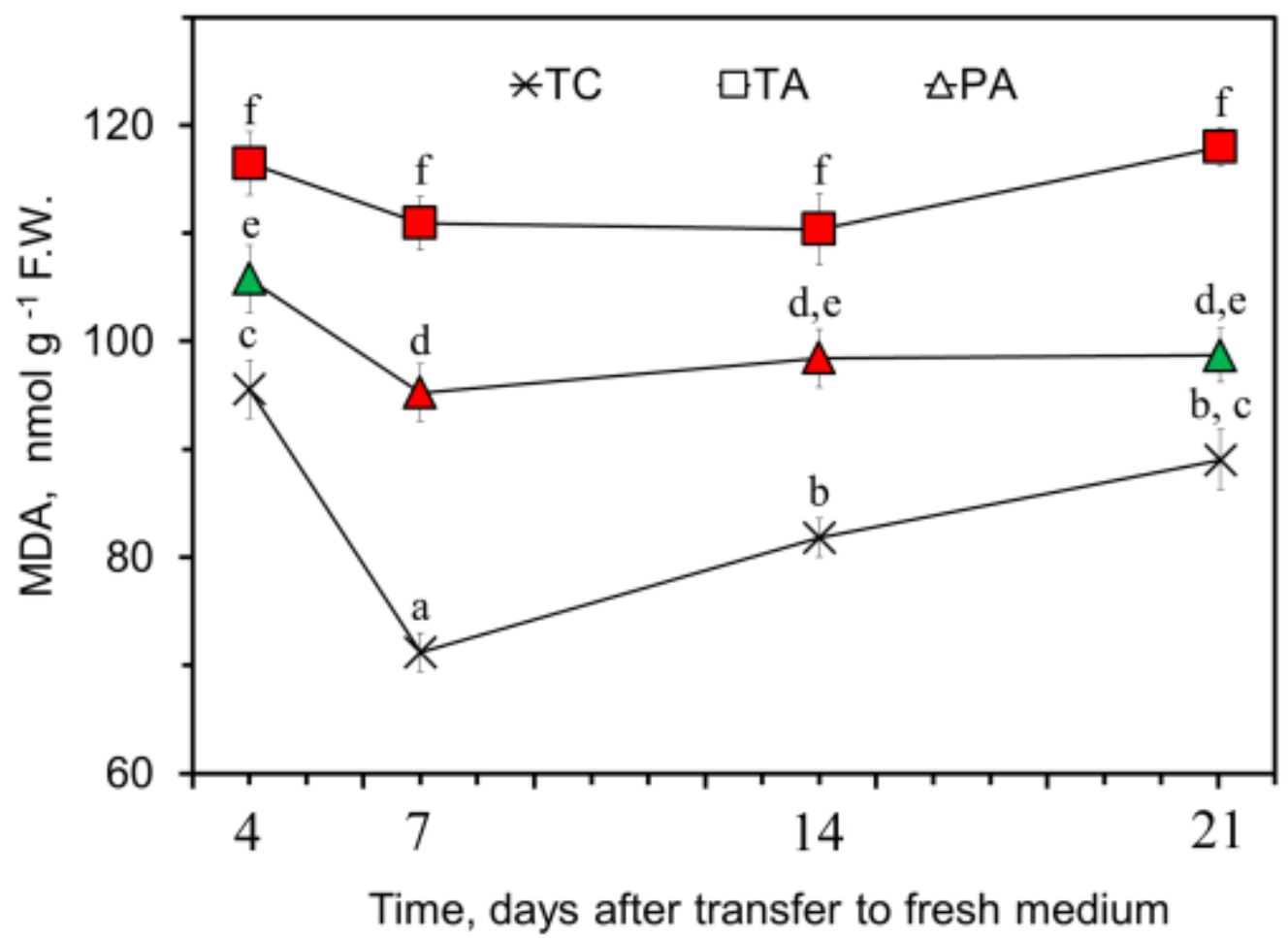

\section{Figure 1}

Effect of antibiotic treatment on MDA accumulation in tobacco in vitro shoots. Control (『), $250 \mathrm{mg} \cdot \mathrm{L}-1$ timentin treated $(\square)$ and post-antibiotic treatment $(\Delta)$ experimental groups were maintained as described in Materials and methods. Data from 4 independent experiments presented as mean \pm standard error. The color of the symbols represents significant differences of the mean values compared to the control at each time point (green $-p<0.05$, red $-p<0.01$ ). Different letters denote significant differences among the time points $(p<0.05)$. 


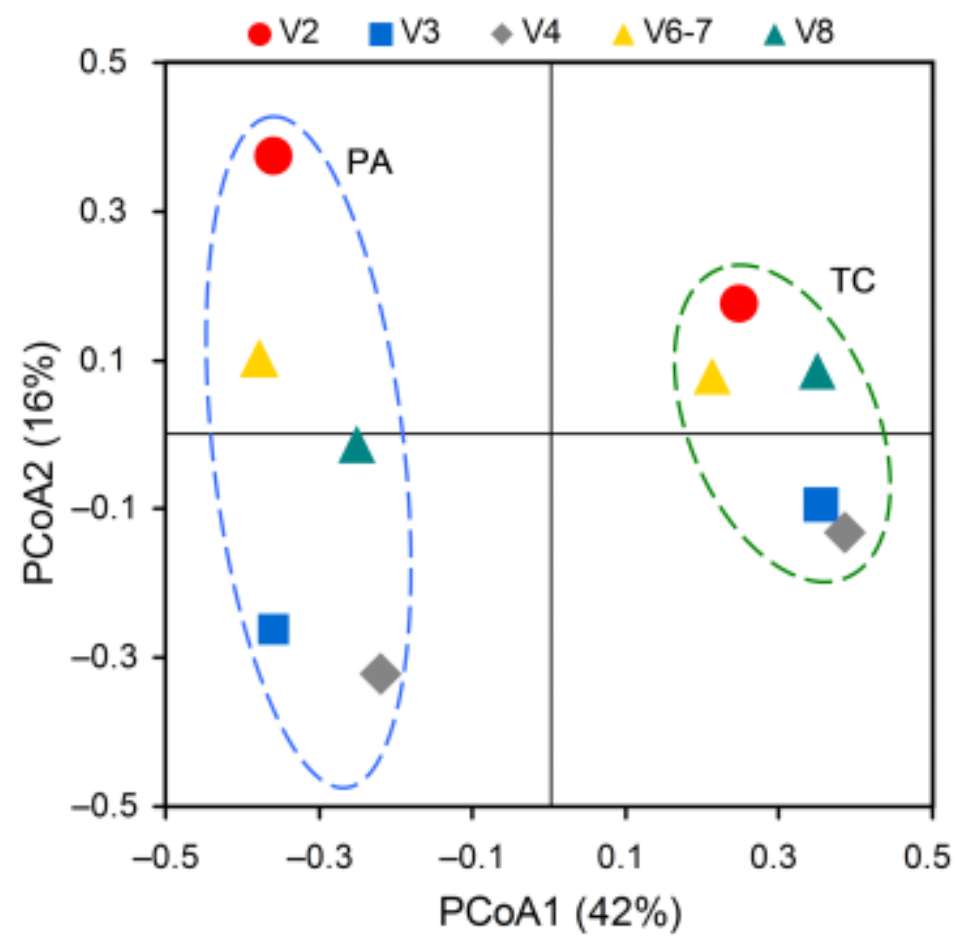

Figure 2

Principal coordinate analysis (PCOA) of variability among the bacterial OTU data sets generated for the tobacco shoot control (TC) and post-antibiotic treatment (PA) samples using five 16S rRNA variable region-specific primer pairs. The analysis was carried out using the Bray-Curtis dissimilarity matrix.

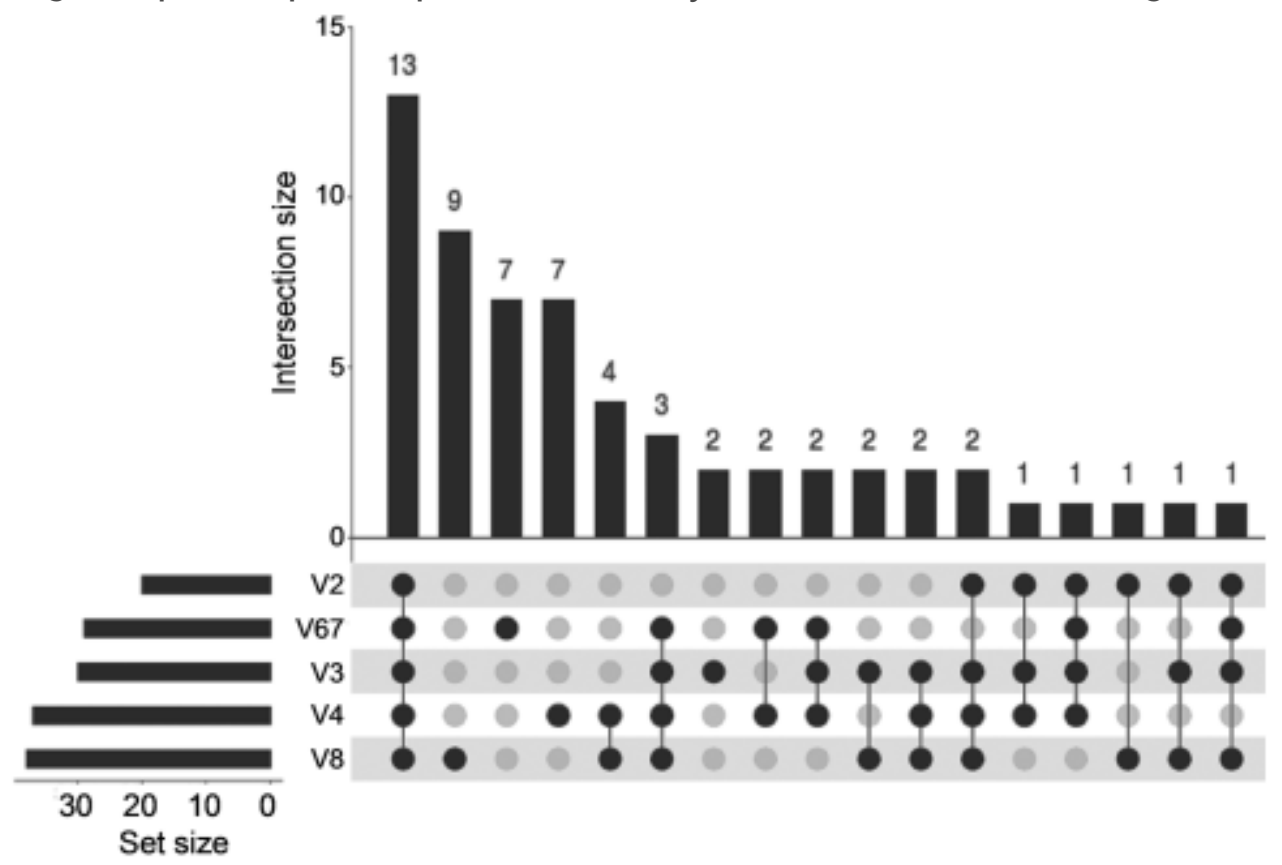

Figure 3

Family level OTU association among data sets generated using five 16S rRNA variable region-specific primer pairs. 


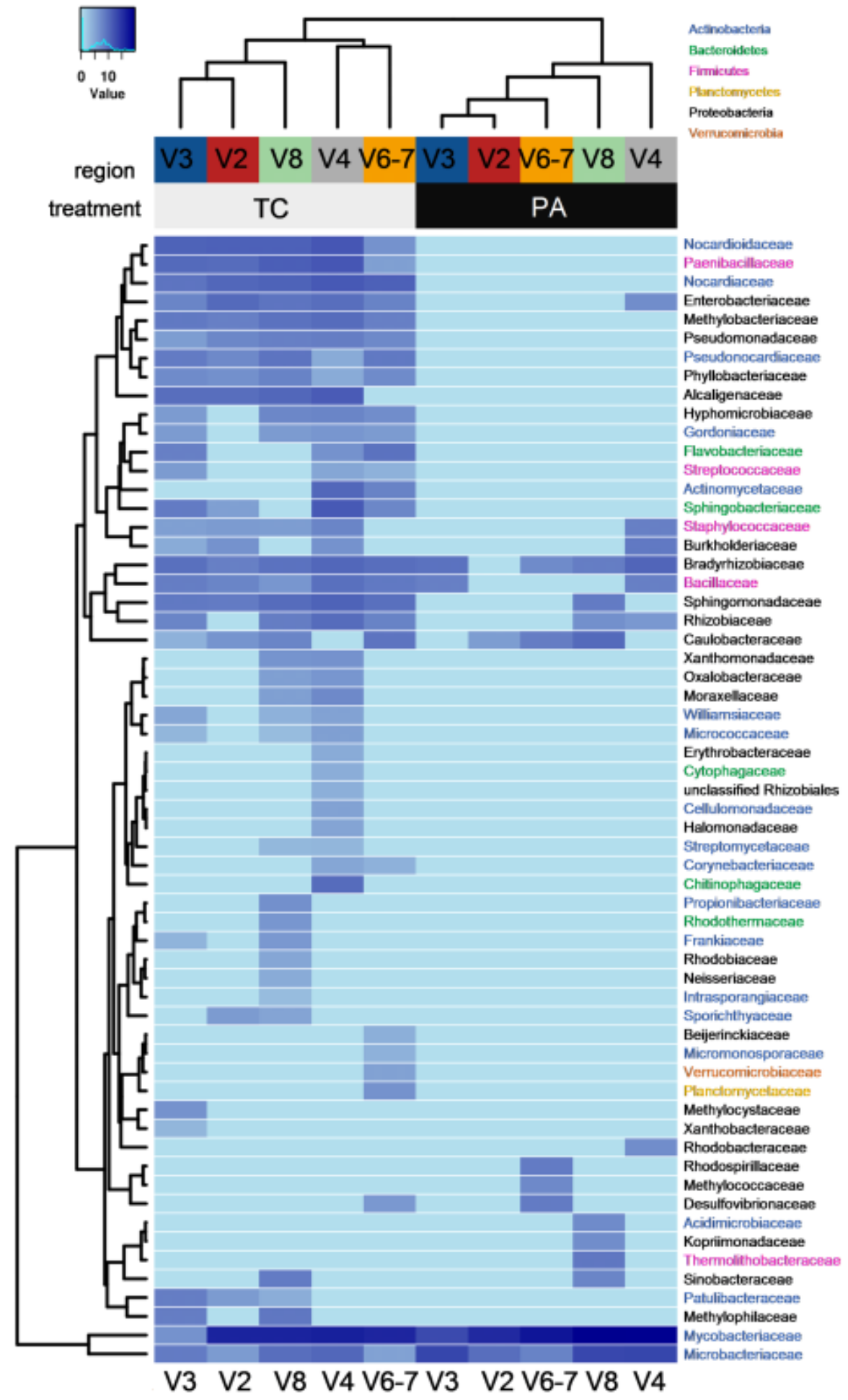

\section{Figure 4}

Family level distribution of reads mapped to high-abundance families generated using five variable $16 \mathrm{~S}$ rRNA region-specific primer pairs for the control (TC) and post-antibiotic treatment (PA) samples of the tobacco in vitro shoots. Different font color represents classes of the Bacteria domain. 

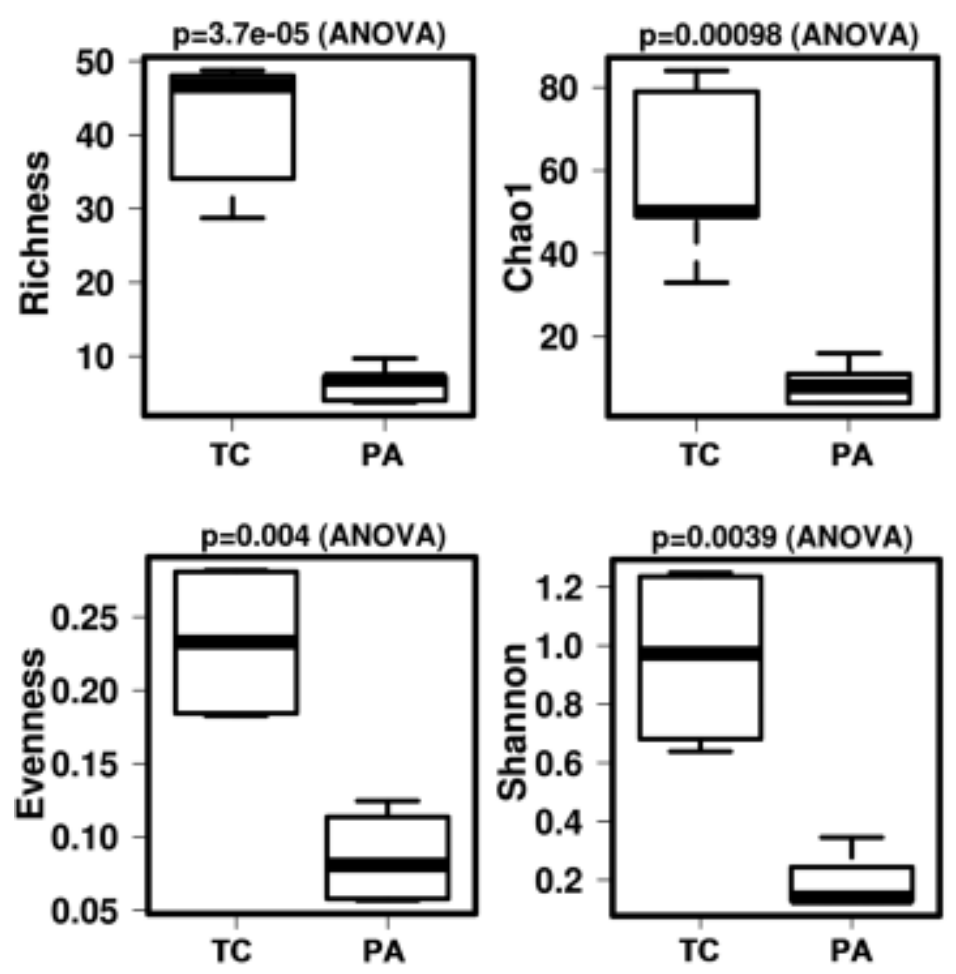

Figure 5

Representative plots of the alpha diversity indices for tobacco in vitro shoot control (TC) and postantibiotic treatment (PA) microbiome data sets. Cumulative data of all five primer pairs was used to estimate Richness and Chao 1 indices, meanwhile data of the V3 region was excluded from the calculation of Evenness and Shannon indices as an outlier.

\section{Supplementary Files}

This is a list of supplementary files associated with this preprint. Click to download.

- SupplementaryinformationTableS3.xls

- Supplementaryinformation.pdf 